\title{
Heimische Pflanzen mit dem Smartphone bestimmen - ein Praxistest
}

\author{
Marco Schmidt \& Hilke Steinecke
}

\section{Abstract}

Since a few years, smartphone apps provide automated identification of plants by artificial intelligence. We have tested three apps free of cost and advertising for identifying 30 species of indigenous plants.

\section{Zusammenfassung}

Smartphone-Apps ermöglichen seit einigen Jahren die automatische Bestimmung von Pflanzen mit Methoden Künstlicher Intelligenz. Wir haben drei werbe- und kostenfrei verfügbare Apps zur Bestimmung 30 heimischer Pflanzenarten getestet.

Seit einigen Jahren gibt es Smartphone-Apps und Websites, die die Bestimmung von Pflanzen durch Bilderkennungssoftware ermöglichen. Waren die Ergebnisse erster Anwendungen auch bei häufigen Arten noch nicht sehr treffsicher, sind mittlerweile die automatischen Bestimmungen deutlich besser geworden und eine echte Hilfe für Laien und Experten gleichermaßen.

Die Technik basiert auf der Auswertung von Mustern in digitalen Bildern durch Convolutional

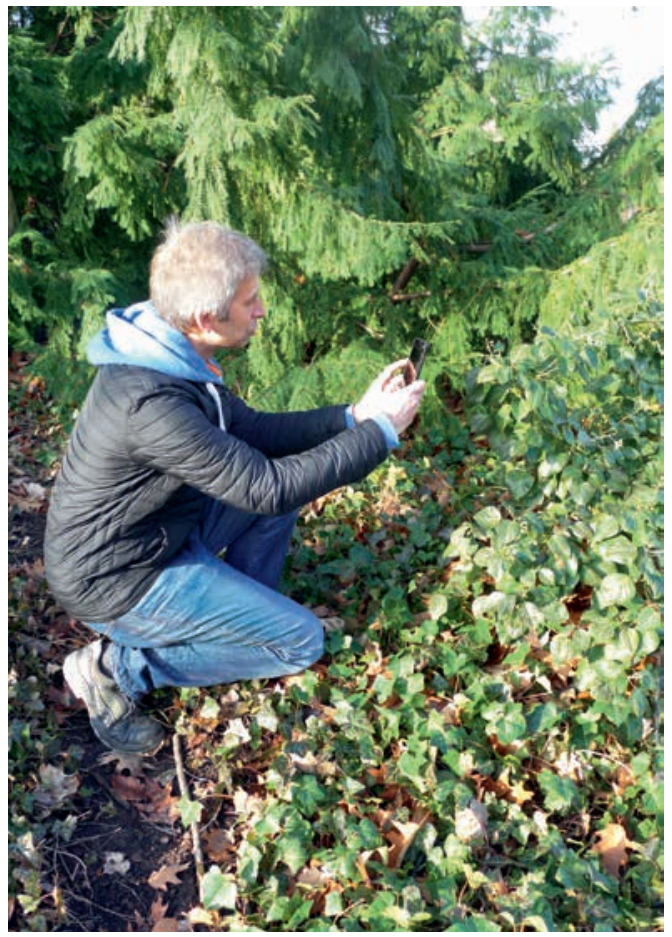

Abb. 1: Mit dem Smartphone im Gelände unterwegs beim Bestimmen von Pflanzen. (Foto: H. Steinecke)
Neural Networks (CNNs), künstliche neuronale Netzwerke, die in ihrer Struktur zu einem gewissen Grad den Nervenstrukturen nachempfunden sind, die die Signale aus unserer Netzhaut verarbeiten und die sehr erfolgreich in zahlreichen Anwendungen zur Bilderkennung eingesetzt werden. Eine wichtige Voraussetzung für eine erfolgreiche Mustererkennung ist ausreichendes Trainingsmaterial, also in unserem Fall Fotos von Pflanzen mit der dazugehörigen Bestimmung. Über Foto- und Bürgerwissenschaftsportale, On-

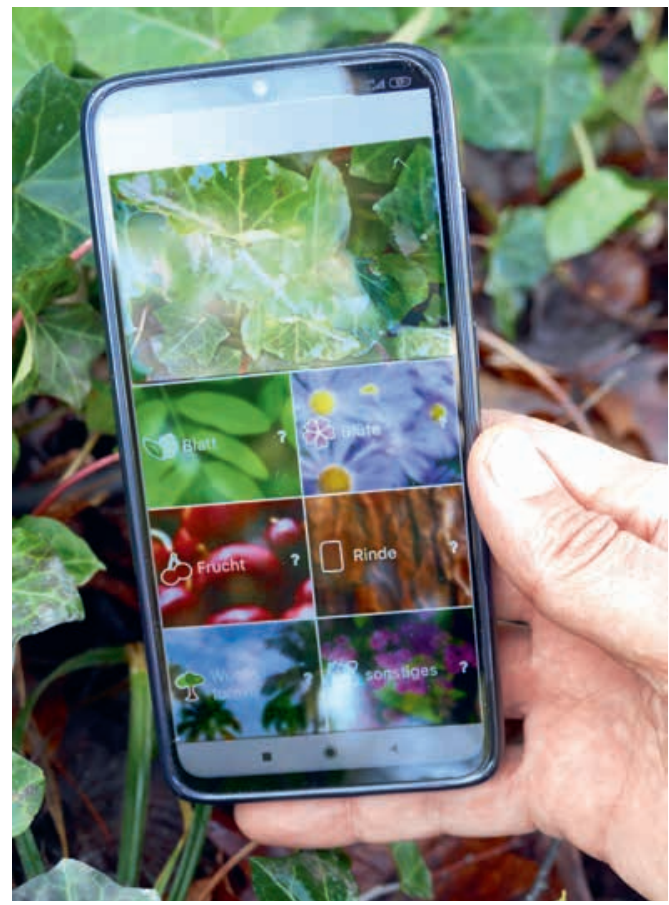

Abb. 2: Blick auf die Bestimmungsapp. Es kann ausgewählt werden, ob die Pflanzen z. B. anhand des Blattes, der Blüte oder der Frucht bestimmt werden sollen. (Foto. H. STEINECKE) 


\begin{tabular}{|c|c|c|c|}
\hline & Flora incognita & Pl@ntnet & iNaturalist \\
\hline Artemisia vulgaris & 0 & 0 & 1 \\
\hline Asplenium scolopendrium & 1 & 1 & 1 \\
\hline Brassica oleracea & 1 & 0 & 1 \\
\hline Calluna vulgaris & 1 & 1 & 1 \\
\hline Claytonia perfoliata & 1 & 1 & 1 \\
\hline Corylus avellana & 1 & 1 & 1 \\
\hline Cymbalaria muralis & 1 & 1 & 1 \\
\hline Cytisus scoparius & 1 & 0 & 1 \\
\hline Dipsacus sativus & 1 & 1 & 1 \\
\hline Euphorbia lathyris & 1 & 1 & 1 \\
\hline Galium odoratum & 1 & 1 & 0 \\
\hline Geranium robertianum & 1 & 1 & 0 \\
\hline Hedera helix & 1 & 1 & 0 \\
\hline Hippophae rhamnoides & 1 & 1 & 1 \\
\hline Ilex aquifolium & 1 & 1 & 1 \\
\hline Juniperus communis & 1 & 1 & 1 \\
\hline Larix decidua & 1 & 1 & 1 \\
\hline Lathyrus vernus & 1 & 1 & 0 \\
\hline Lunaria rediviva & 1 & 0 & 0 \\
\hline Matricaria chamomilla & 0 & 1 & 0 \\
\hline Alkekengi officinarum & 0 & 0 & 0 \\
\hline Pinus mugo & 0 & 1 & 0 \\
\hline Polypodium vulgare & 1 & 1 & 1 \\
\hline Ruscus aculeatus & 1 & 1 & 1 \\
\hline Ruta graveolens & 1 & 1 & 1 \\
\hline Salvia rosmarinus & 0 & 1 & 1 \\
\hline Solanum dulcamara & 0 & 0 & 0 \\
\hline Taxus baccata & 1 & 1 & 1 \\
\hline Teucrium scorodonia & 1 & 0 & 1 \\
\hline Vinca minor & 1 & 1 & 1 \\
\hline Erkennungsquote & $80.00 \%$ & $76.67 \%$ & $70.00 \%$ \\
\hline
\end{tabular}

Tabelle 1: Bestimmungserfolg verschiedener Smartphone-Apps an 30 heimischen Pflanzenarten. 
linefloren und vergleichbare digitale Ressourcen sind im vergangenen Jahrzehnt umfangreiche Trainingsdaten entstanden, es wird aber oft auch auf speziell für die jeweilige Anwendung erstellte Trainingsdatensets zurückgegriffen.

Wir haben drei werbe- und kostenfrei angebotene Smartphone-Apps ausgesucht und Mitte Dezember 2019 an 30 heimischen Pflanzenarten im Botanischen Garten Frankfurt getestet:

- Flora Incognita wird an der TU Ilmenau und dem MPI Jena mit einem Fokus auf mitteleuropäischer Flora entwickelt. Außer Bildern werden auch Beobachtungsdatum und Geografie in den Bestimmungsalgorithmus einbezogen.

-Pl@ntnet ist eine der ersten Anwendungen zur automatischen Pflanzenerkennung, entwickelt durch ein Konsortium französischer Forschungsinstitute. Die App ist auf zahlreiche Wild-, Zier- und Nutzpflanzen aus der ganzen Welt trainiert.

- iNaturalist ist keine Bestimmungs-App, sondern eine globale Bürgerwissenschaftsplattform für Naturbeobachtungen, die dem Nutzer aber beim Hochladen KI-basierte Bestimmungen vorschlägt und andere Bestimmungshilfen bietet.

Es gibt einige Unterschiede in der Anwendung, so wird man in Flora Incognita beispielsweise aufgefordert, bestimmte Details/Ansichten einer Pflanze zu fotografieren, soll diese auch in Pl@ntnet angeben, während dies bei iNaturalist keine Rolle spielt. Die Ergebnisanzeige ist auch verschieden, so werden oft mehrere Optionen angezeigt, teils mit Angaben, wie „sicher" die Bestimmung sei. Zum Vergleich haben wir nur die jeweils ersten Ergebnisse verglichen, unabhängig davon, ob diese als „sicher bestimmt" angegeben wurden.

Unser Test erfolgte zu einer für Pflanzenbeobachtungen ungünstigen Jahreszeit. Wir haben Pflanzen ausgesucht, die aus unserer Sicht noch gut erkennbar waren und nicht zu schwierigen Gruppen mit zahlreichen Kleinarten o.ä. gehören. Dennoch wurden etwa der Bittersüße
Nachtschatten oder die Lampionblume, bei denen keine Blätter mehr vorhanden waren, von keiner der Apps als Erstergebnis korrekt bestimmt. Alle Apps schneiden insgesamt mit 70\% bis $80 \%$ korrekten Bestimmungen aber erstaunlich gut ab, insbesondere, wenn man berücksichtigt, dass bei Bestimmungen mit einem abweichenden Ergebnis die korrekten Bestimmungen stets unter den ersten fünf Ergebnissen zu finden waren.

Wir können alle genannten Apps für eine einfache und schnelle Bestimmung empfehlen. Vor allem dem Anfänger sind solche Apps sicher eine Hilfe und ein guter Einstieg, insbesondere wenn die Zuordnung zu einer Verwandtschaftsgruppe ebenso unbekannt ist wie das in Bestimmungsschlüsseln verwendete Fachvokabular. Man sollte sich nur nicht dazu verleiten lassen, das Bestimmungsergebnis allzu unkritisch zu akzeptieren, sondern lieber mit einem merkmalsbasierten Schlüssel oder anhand von Bildmaterial überprüfen, ob die Bestimmung plausibel ist. Experten werden vor allem von BestimmungsApps profitieren, wenn ihnen die Einordnung in eine Verwandtschaftsgruppe schwerfällt oder ein Name einfach nicht einfallen will. Mit einer sich stetig verbessernden Basis an Trainingsdaten ist aber davon auszugehen, dass in nicht allzu ferner Zukunft auch schwierigere Gruppen und seltenere (und daher bislang schlechter fotografisch dokumentierte) Arten besser per KI bestimmt werden können.

\section{Internetseiten}

https://floraincognita.com

https://plantnet.org/

https://www.inaturalist.org

\section{Anschriften des Autors und der Autorin}

Dr. Marco Schmidt, Palmengarten Frankfurt, Siesmayerstr. 61, 60323 Frankfurt,

E-Mail: marco.schmidt@stadt-frankfurt.de

Dr. Hilke Steinecke, Palmengarten Frankfurt, Siesmayerstr. 61, 60323 Frankfurt,

E-Mail: hilke.steinecke@stadt-frankfurt.de 